\title{
Genome sequencing of a yeast-like fungal strain P6, a novel species of Aureobasidium spp.: insights into its taxonomy, evolution, and biotechnological potentials
}

\author{
Shu-Lei Jia ${ }^{1} \cdot$ Yan $\mathrm{Ma}^{1} \cdot$ Zhe Chi $^{1} \cdot$ Guang-Lei Liu ${ }^{1} \cdot$ Zhong Hu$^{2} \cdot$ Zhen-Ming Chi $^{1}$
}

Received: 2 August 2019 / Accepted: 4 November 2019/Published online: 6 December 2019

(C) The Author(s) 2019

\begin{abstract}
Purpose This study aimed to look insights into taxonomy, evolution, and biotechnological potentials of a yeast-like fungal strain P6 isolated from a mangrove ecosystem.

Methods The genome sequencing for the yeast-like fungal strain P6 was conducted on a Hiseq sequencing platform, and the genomic characteristics and annotations were analyzed. The central metabolism and gluconate biosynthesis pathway were studied through the genome sequence data by using the GO, KOG, and KEGG databases. The secondary metabolite potentials were also evaluated.

Results The whole genome size of the P6 strain was $25.41 \mathrm{Mb}$ and the $\mathrm{G}+\mathrm{C}$ content of its genome was $50.69 \%$. Totally, 6098 protein-coding genes and 264 non-coding RNA genes were predicted. The annotation results showed that the yeast-like fungal strain P6 had complete metabolic pathways of TCA cycle, EMP pathway, pentose phosphate pathway, glyoxylic acid cycle, and other central metabolic pathways. Furthermore, the inulinase activity associated with $\beta$-fructofuranosidase and high glucose oxidase activity in this strain have been demonstrated. It was found that this yeast-like fungal strain was located at root of most species of Aureobasidium spp. and at a separate cluster of all the phylogenetic trees. The P6 strain was predicted to contain three NRPS gene clusters, five type-I PKS gene clusters, and one type-I NRPS/PKS gene cluster via analysis at the antiSMASH Website. It may synthesize epichloenin A, fusaric acid, elsinochromes, and fusaridione A.

Conclusions Based on its unique DNA sequence, taxonomic position in the phylogenetic tree and evolutional position, the yeastlike fungal strain P6 was identified as a novel species Aureobasidium hainanensis sp. nov. P6 isolate and had highly potential applications.
\end{abstract}

Keywords Aureobasidium spp. An undescribed species · Genome $\cdot$ Glucose oxidase $\cdot$ Phylogenetic tree $\cdot$ Evolutional position

\section{Introduction}

The genus ascomycetous Aureobasidium spp. are black yeastlike fungi of the family Saccotheciaceae within the class of the Dothideomycetes. So far, A. microstictum, A. proteae, A. pullulans, A. lini, A. namibiae, A. melanogenum,

Electronic supplementary material The online version of this article (https://doi.org/10.1007/s13213-019-01531-1) contains supplementary material, which is available to authorized users.

Zhen-Ming Chi

chi@ouc.du.cn

1 College of Marine Life Sciences, Ocean University of China, Yushan Road, No. 5, Qingdao, China

2 Department of Biology, Shantou University, Shantou 515063, China
A. leucospermi, A. subglaciale, A. iranianum, A. caulivorum, A. mangrovei, A. thailandense, A. pullulans var. aubasidan, A. pini, and A. khasianum have been obtained and classified (Nasr et al. 2018; Jiang et al. 2019; Prabhugaonkar and Pratibha 2018). At the same time, the genomic DNAs of many strains of the genus have been sequenced and the sequenced DNAs have been annotated (Gostinčar et al. 2014; Zhao et al. 2019; Chan et al. 2012). However, it is still completely unknown about their genome evolution. It has been known that different strains with high genetic variations of the genus are widely distributed in the world, including soils, water, the phylloplane, wood, and many other plant materials, rocks, monuments, limestone, hypersaline habitats, coastal water, deep sea, marine sediments of Antarctica, desert, natural honey, and mangrove systems, demonstrating that it is an ubiquitous and widespread genus 
and can adapt to various environments, including many harsh surroundings (Jiang et al. 2016a). However, the reason for its widespread distribution is also still unclear. This may be due to high genetic variations of different strains isolated from different environments and high adaptation ability. Especially, these yeast-like fungi are largely known as sources of commercial pullulan, polymalate, liamocin, intracellular lipids, gluconic acid, siderophore, melanin, and various enzymes (Ma et al. 2014; Chi et al. 2016; Garay et al. 2018; Wang et al. 2014; Ma et al. 2018; Chi et al. 2012; Li et al. 2007; Ni et al. 2008; Liu et al. 2008; Chen et al. 2017a; Aung et al. 2019; Jiang et al. 2016a). This means that different strains of the genus have many potential applications in basic research and biotechnology (Prasongsuk et al. 2018). Therefore, it is very important to acquire more strains of Aureobasidium spp. from different environments.

In our previous studies (Ma et al. 2018), it was found that the yeast-like fungal strain P6 isolated from a mangrove ecosystem can produce a large amount of $\mathrm{Ca}^{2+}$-gluconic acid (GA) from glucose and $\mathrm{CaCO}_{3}$ because its high glucose oxidase activity. It also has been shown that the yeast-like fungal strain P6 has a $\beta$-fructofuranosidase with a high inulinhydrolyzing activity (Jiang et al. 2016b). However, its taxonomic position, evolution, and more biotechnological potentials are still unclear. So in this study, its genomic DNAs were sequenced and the yeast-like fungus taxonomy, evolution, and biotechnological potentials were analyzed based on the sequenced DNA.

\section{Materials and methods}

\section{Yeast-like fungal strain, media, and cell growth}

The yeast-like fungal strain P6 used in this study was isolated from the mangrove system (the leaf of the mangrove plant, Kandelia candel and Latitude and longitude of the sampling site at DongZaiGou, Haikou, Hainan Province, China are $\mathrm{N} 19^{\circ} 53^{\prime} \mathrm{E} 110^{\circ} 1^{\prime}$ ) (Ma et al. 2013) and was found to be able to produce high level of $\mathrm{Ca}^{2+}-\mathrm{GA}$ and a $\beta$ fructofuranosidase with a high inulin-hydrolyzing activity (Ma et al. 2018; Jiang et al. 2016b). The media for cell growth were a YPD medium containing $20.0 \mathrm{~g} / 1$ glucose, $20.0 \mathrm{~g} / \mathrm{l}$ polypepton, and $10.0 \mathrm{~g} / 1$ yeast extract and a potato-dextroseagar (PDA) medium with $200.0 \mathrm{~g} / 1$ potato extract and $20.0 \mathrm{~g} / 1$ glucose. It was grown on the YPD plate and the PDA plate with $20.0 \mathrm{~g} / 1$ agar at $30^{\circ} \mathrm{C}$ for 4 days, respectively.

\section{Genomic DNA isolation of the yeast-like fungal strain P6}

For DNA isolation, the yeast-like fungal strain P6 was aerobically grown in $5.0 \mathrm{ml}$ of the liquid YPD medium at $28^{\circ} \mathrm{C}$ and $180 \mathrm{rpm}$ for $10-12 \mathrm{~h}$ and the yeast-like fungal cells in $500.0 \mu \mathrm{l}$ of the culture were harvested and washed with sterile distilled water by centrifugation at $5000 \times g$ and $4{ }^{\circ} \mathrm{C}$ for $5 \mathrm{~min}$. The DNA in the washed cells was then isolated according to the protocol described by Chi et al. (2012). The integrity, purity, and quantity of the DNA (total amount of DNA was $6.0 \mu \mathrm{g}, \mathrm{OD}_{260 / 280 \mathrm{~nm}}=1.8-2.0$ )were spectrophotometrically evaluated with a spectrophotometer.

\section{Yeast-like fungal strain identification}

Fermentation and metabolic characterization of the yeast-like fungal strain P6 were carried out based on the procedures as described by Kurtzman and Fell (2000). Molecular identification was conducted accordingly with amplification of the internal transcribed spacer (ITS) (Supplementary file 1) region and 28S rDNA (the accession number: KF260961) and the genes encoding elongation factor- $1 \alpha$ and $\beta$-tubulin, followed by the DNA sequencing. The identity of the yeast-like fungal isolate was determined via BLASTn search against NCBInucleotide database. A total of 33 ITS sequences of all the Aureobasidium spp. (ITS regions of A. melanogenum P16 and A. pullulans $\mathrm{P} 25$ are sequenced in our laboratory) together with a representative strain from other studies and three outgroup strains, Sydowia polyspora, Aspergillus aculeatus, and Saccharomyces cerevisiae were obtained from GenBank to construct a phylogenetic tree (Supplementary file 1).

The genome-wide phylogenetic tree based on the genomes of the yeast-like fungal strain P6 and other Aureobasidium spp. strains was constructed using a TreeBeST (Nandi et al. 2010) and the method of a PhyML with the setting bootstraps of 1000. All the accession numbers of the genomes from the yeast-like fungal strains used in this study are listed in Supplementary file 2.

The phylogenetic tree of the yeast-like fungal strain P6 and other strains of Aureobasidium spp. based on a multigene analysis of the ITS sequences, 28S rDNA, EF- $1 \alpha$ and $\beta$ tubulin obtained from their genomic DNAs (Zalar et al. 2008) were also constructed by the neighbor-joining method using a MEGA 7.0.14 software (Kumar et al. 2016), Bootstrap values (1000 pseudoreplications) were $\geq 71 \%$, and Sydowia polyspora strain CBS544.95 was used as the outgroup.

\section{Genome sequencing and de novo assembly}

The genomic DNA was further purified using SDS methods. One paired-end library with an input fragment size of 500-bp and one mate-pair library with $5.0-\mathrm{kb}$-insert size were constructed using a Next Ultra DNA Library Prep Kit for Illumina (New England Biolabs Ltd). Quality and concentration of the libraries were checked using an Agilengt 2100 Bioanalyzer (Agilent Technologies, Palo Alto, CA, USA) and a Qubit2.0 Fluorescence Spectrophotometer (Invitrogen, 
Carlsbad, CA, USA) in order to discard adapter reads and lowquality reads. Subsequently, the libraries with high-quality reads were sequenced using a HiSeq 2500 sequence platform provided by the Illumina Company and PE125 strategies to obtain Raw Data. The Raw Data with the low quality were filtrated with SOAPnuke v.1.5.6 (SOAPnuke, RRID: SCR_015025) (Chen et al. 2017b) to obtain Clean Data. The Clean Data were assembled at a value $k=50$ using Vector NTI Advance 9.1.0, Contig Express, and SEQUENCER 4.6 to acquire a raw scaffold. The gap in the raw scaffold was complemented using a GapCloser software, and the raw scaffold was optimized to discard the DNA fragment less than $500 \mathrm{bp}$ to obtain the final scaffold. Preliminary assembly of the filtered valid data was done using SOAPdenovo2 (v.2.04.4; SOAPdenovo2, RRID: SCR 014986) (Li et al. 2010), and then the Krskgf (v. 1.19, https://github.com/ gigascience/paper-zhang2014), GapCloser (v.1.10) (Luo et al. 2012), and other programs were used to optimize the preliminary assembly results and improve the assembly effect. The draft genome data were finally deposited on GenBank (accession number: RZIQ01000000).

\section{Gene prediction and annotation}

Gene prediction of the draft genome of the yeast-like fungal strain P6 was carried out using a GeneMarkS v.4.28 (http:// topaz.gatech.edu/GeneMark/) (Besemer et al. 2001) with an integrated model which combined the GeneMarkS generated (native) and Heuristic model parameters. The function of putative coding sequences (CDSs) was annotated via local BLAST searches against NCBI NR and SwissProt databases. Then, the genome blast search (Altschul et al. 1990) ( $E$ value less than $1 * \mathrm{e}^{-5}$, minimum alignment length $\geq 40 \%$, matching similarity $\geq 40 \%$ ) was performed against six databases, including Kyoto Encyclopedia of Genes and Genomes (KEGG) (http://www.kegg.jp/kegg/tool/annotate_sequence. html) for the metabolic pathways (Kanehisa et al. 2004), NCBI-NR (Non-Redundant Protein Database, ftp://ftp.ncbi. nih.gov/blast/db/FASTA/nr.gz) for protein alignments, the SwissProt (ftp://ftp.ebi.ac.uk/pub/databases/uniprot/ knowledgebase/uniprot_sprot.fasta.gz) for mapping the gene-ontology terms (Magrane and Consortium 2011), KOG (Eucaryotic Orthologous Groups) (Koonin et al. 2004) for eukaryotic clusters of orthologues, and Gene Ontology (GO, http://www.geneontology.org/) for annotation of the homologous genes and their function, location of cellular components, and biological processes. The antiSMASH website (http://antismash.secondarymetabolites.org/) was used to predict the gene cluster encoding yeast-like fungal strain secondary metabolites and metabolic pathways (Blin et al. 2013). Predicted protein models were submitted to a dbCAN2 meta-server (Zhang et al. 2018) for annotation of carbohydrate-active enzymes (CAZymes). The prediction of secreted proteins was carried out using the method of Ohm et al. (2012). The organization of putative gene clusters were retrieved from the genome using a sequence viewer Artemis version 12.0 (Rutherford et al. 2000).

The BLAST and ORF Finder programs at the National Center for Biotechnology Information (NCBI) were used for the nucleotide sequence analysis, deduction of the amino acid sequence from different genes (Cañete-Rodríguez et al. 2016).

Circular representation of the complete genome of the yeast-like fungal strain P6 including GC content and ORFs was built with a CGView Server (Grant and Stothard 2008). The CGView Server is an online server that keeps using this name with no changes or updated version.

\section{Analysis of orthologous genes and unique genes}

We analyzed the pan-genome and searched for orthologous genes in the genome of different Aureobasidium spp. strains with the software package of GET_HOMOLOGUES v3.2.1 (Contrerasmoreira and Vinuesa 2013), which could cluster homologous gene families using the bidirectional best-hit, COGtriangles, or OrthoMCL v2.0 clustering algorithms (Contrerasmoreira and Vinuesa 2013).

\section{The divergency time analysis}

The ITS sequences of different species are conservative in the evolutionary process. Therefore, the ITS sequences in different species of Aureobasidium spp. were selected to estimate the divergence time. The ITS sequences were aligned using a MAFFT v7.149 (Katoh et al. 2002) and automatically trimmed by a trimAl v3.0 (Capella-Gutiérrez et al. 2009). These analyses were performed using the BEAST v1.10.1 software package (Suchard et al. 2018) to estimate the divergence time based on the ITS sequences of different species of Aureobasidium spp. (Garnica et al. 2016).

We chose the estimated divergence time of 590 Mya of $S$. cerevisiae and A. aculeatus on the Website of TimeTree of Life (http://www.timetree.org/). Besides that, the GTR + $\mathrm{G}$ model was chosen as the best substitution model through the Website of a ATGC:PhyML 3.0 (http://www.atgcmontpellier.fr/phyml/). The strict molecular clock model and the constant size coalescent prior set were used to estimate the divergence time and the corresponding credibility intervals. Tree prior was set to Yule speciation. BEAST analyses were run for 50 million generations, logging parameters and trees every 1000 generations. Convergence, mixing, and effective sample sizes (ESS) of parameters were checked using a Tracer v1.7.1 (Rambaut et al. 2018). A FigTree v1.4.3 was used to visualize the resulting tree and obtain the means and $95 \%$ higher posterior densities (HPD) (http://tree.bio.ed.ac.uk/ software/figtree/). 


\section{Amino acid sequence alignment and conservative domain analysis}

A DNAMAN v9.0.1.116 (https://www.lynnon.com/index. html) was used for amino acid sequence alignment and conservative domain analysis. A BioEdit v7.0.9.0 (http:// www.mbio.ncsu.edu/bioedit/bioedit.html) was used to make the comparison of homologous genes and search the regulation genes of the metabolic pathway with a software UltraEdit v25.00.0.58 (https://www.ultraedit.com/). The protein domain alignment and analysis were conducted at the NCBI-Blast Website (https://blast.ncbi.nlm.nih.gov/Blast. cgi).

\section{Selection pressure analysis of genes}

Selective pressure analysis was conducted with a PAML 4.9 (Yang 2007). The selection pressure of genes in evolutionary process was analyzed by comparing the omega $(\mathrm{dN} / \mathrm{dS})$ values of different branch lengths with a NSsites set as a single parameter model.

\section{Genome collinearity analysis}

Based on the results of the protein blastp comparisons of different strains of Aureobasidium spp. and the location of these genes in the genome, synteny from paralogs and orthologs was detected by using a MCScanX (the MCScanX is an upgraded version of MCScan without any new version) (Wang et al. 2012). Finally, we visualized and analyzed the collinear genes located on different scaffolds combined with the software TBTools.

\section{Analysis of promoter and transcription factors}

The YEASTRACT Website (http://www.yeastract.com/index. php) provides a convenient way to search for transcription factors and analyze the transcription factor binding sites (Teixeira et al. 2006). Besides that, we predicted transcription regulation networks in yeast from the data emerging from gene-by-gene analysis or global approaches. The possible transcription promoters were found through the website of Berkeley Drosophila Genome Project (BDGP) (http:/www. bdgp.org/seq tools/promoter.html).

\section{Prediction of the secondary metabolite gene clusters NRPSs and PKSs}

The online Website antiSMASH (https://fungismash. secondarymetabolites.org/) (Blin et al. 2013; Weber et al. 2015) was used to predict the secondary metabolite gene clusters NRPSs and PKSs in the yeast-like fungal strain P6 genome.

\section{Results and discussion}

\section{The P6 strain genome content and structure}

The yeast-like fungal strain P6 draft genome sequence (Supplementary file 2) was generated using the Illumina platform. Table 1 shows that 24,636,141 bp were assembled into 240 gap-free scaffolds ranging from $0.5 \mathrm{~kb}$ to $0.69 \mathrm{Mbp}, 303$ contigs ranging from $0.5 \mathrm{~kb}$ to $0.575 \mathrm{Mbp}$, and the $\mathrm{G}+\mathrm{C}$ content of its genome was $50.69 \%$. Gene predictions identified 6098 putative protein-coding genes (CDS) that made up $34.13 \%$ of the whole genome sequence which contained 264 ncRNAs including 204 tRNAs, 41 5SrRNAs, 2 sRNAs, and 17 snRNAs. The $\sim 24-\mathrm{Mb}$ of the P6 strain genome was smaller in size than those of other strain genomes of Aureobasidium spp. with published genome sequences of A. pullulans P25 (30.97 Mbp) (Zhao et al. 2019), A. pullulans (29.62 Mbp), A. melanogenum (26.2 Mbp), A. subglaciale (25.8 Mbp), A. namibiae (25.43 Mbp) (Gostinčar et al. 2014), and A. pullulans AY4 (26.72 Mbp) (Chan et al. 2012) (Table 1; Fig. 1a), but they had the similar $\mathrm{G}+\mathrm{C}$ content of around $50 \%$. This meant that the strain P6 draft genome was different from that of any other strains of Aureobasidium spp. The secondary metabolite-encoding gene clusters were predicted by submitting the whole genome sequence in fasta formula to the antiSMASH Website. Table 1 also showed that the whole

Table 1 General characteristics of the yeast-like fungal strain P6 genome

\begin{tabular}{ll}
\hline General features & The yeast-like fungal strain P6 \\
\hline Length (bp) & $24,636,141$ \\
Scaffolds & 240 \\
GC content (\%) & 50.69 \\
Protein-coding gene number & 6098 \\
Coding region of genome (\%) & 34.13 \\
Total number of predicted ORFs & 6098 \\
tRNA & 204 \\
5SrRNA & 41 \\
sRNA & 2 \\
snRNA & 17 \\
Properties of predicted gene models & No. of genes \\
KEGG alignment & 2719 \\
KOG assignment & 3334 \\
GO assignment & 2162 \\
NOG assignment & 5199 \\
SwissProt assignment & 2427 \\
NR assignment & 5646 \\
T1pks-Nrps & 1 \\
Nrps & 3 \\
T1pks & 5 \\
\hline
\end{tabular}




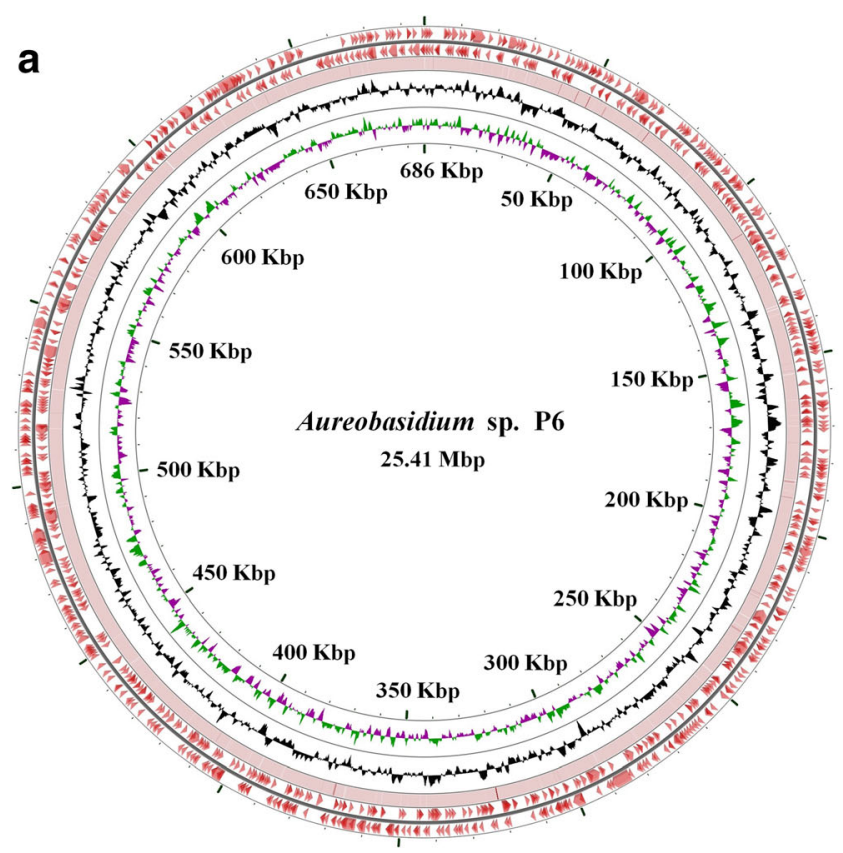

Fig. 1 a Circular representation of the yeast-like fungal strain P6 complete genome. Circles (from inside to outside): circle 1, GC skew; circle 2, GC content; circle 3, blast hits by reading frame; circles 4 and 5 , (ORFs). The CGView Server (http://stothard.afns.ualberta.ca/cgview_

genome of the P6 strain contained three NRPS clusters, five type-I PKS clusters, and one NRPS/PKS cluster.

The identification of orthologs shared in different strains of Aureobasidium spp. using the core-pan homologous gene indicated that out of the predicted 6098 putative protein-coding sequences (CDS) for the P6 strain, 2135 genes were unique to the P6 strain genome for this particular comparison with the genome of A. melanogenum P16, a high pullulan-producing yeast-like fungal strain (Supplementary file 2) (Ma et al. 2014) and 1994 genes were the P6 strain genome specific compared with the genome of $A$. pullulans $\mathrm{P} 25$, also a high gluconic acid-producing yeast (Zhao et al. 2019) while the remaining 23\% (1408 genes) had detectable unique genes in the four genomes of Aureobasidium spp. (Fig. 1b). The annotation of the unique 1408 genes using KEGG revealed that they were involved in 275 metabolism pathways which could be divided into three categories: (1) were implicated with DNA repair and recombination, biosynthesis of ribosome and tRNA; (2) took part in glycosylation and biosynthesis of cytochrome P450, peroxisome, and lysosome; and (3) signal transduction (Fig. 2). Therefore, they mainly were responsible for translation, cellular cycle (MAPK signal pathways), and metabolisms of some amino acids (Fig. 2). Among the annotated genes, the specific 148 genes of the P6 strain encoded acid protease (KEQ87861.1), $\mathrm{Ca}^{2+} / \mathrm{H}^{+}$antiporter (KEQ62719.1), DNArepairing proteins (KEQ80820.1 and KEQ66757.1), glutathione $S$-transferases (KEQ80961.1), P450 cytochrome monoxygenase (KEQ88789.1), Cu/Zn superoxide dismutase (KEQ80108.1), oxidoreductase (KEQ62889.1), FMN-

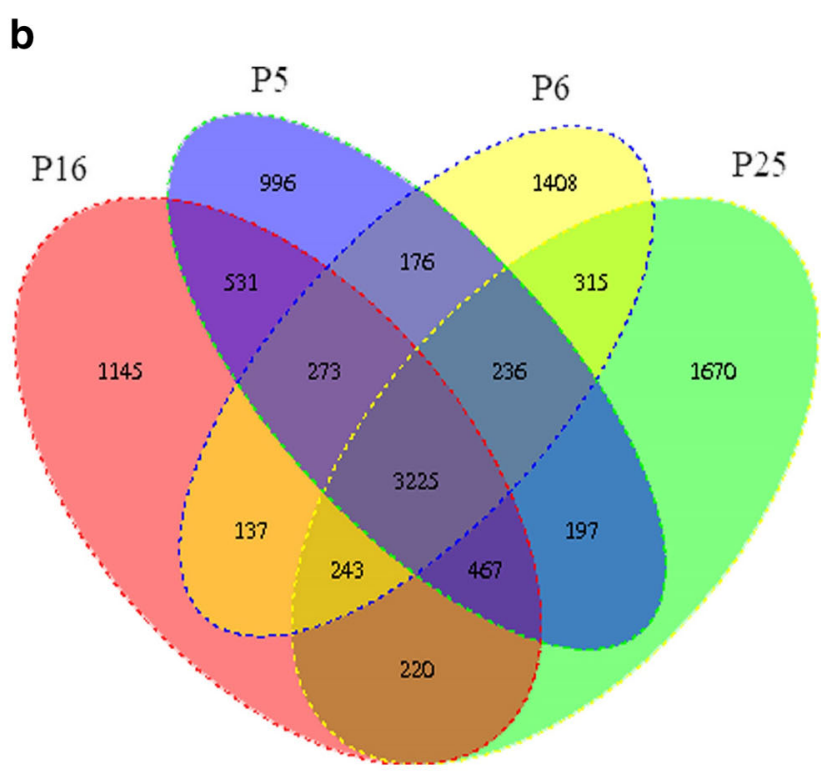

server/) was used to build the circular representation. Mapping studies were done using BLASTn with an $E$ value cut-off $1 \mathrm{e}^{-5}$. b The shared and unique genes of the genomes of A. melanogenum P16 and P5, A. pullulans P25 and the yeast-like fungal strain P6

oxidoreductase (KEQ76182.1), and FAD/NAD(P)-binding domain proteins (KEQ59632.1 and KEQ78682.1), suggesting that they could play an important role in antioxidant activity in the cells.

\section{The P6 strain was a new species of Aureobasidium spp.}

As stated in the "Introduction" section, Aureobasidium spp. can be divided into A. microstictum, A. proteae, A. pullulans, A. lini, A. namibiae, A. melanogenum, A. leucospermi, A. subglaciale, A. iranianum, A. caulivorum, A. mangrovei, A. thailandense, A. pullulans var. aubasidan, A. pini, and A. khasianum (Nasr et al. 2018; Jiang et al. 2019; Prabhugaonkar and Pratibha 2018). However, the results in Fig. 3a-d clearly demonstrated that the sequences of ITS, $28 \mathrm{~S}$ rDNA, the genes encoding $\beta$-tubulin, and elongation factor 1 (EF1) from the P6 strain were far related to those of ITS, 28S rDNA, the genes encoding $\beta$-tubulin, and EF1 from any other species of Aureobasidium spp. For example, the similarities between ITS, $28 \mathrm{~S}$ rDNA, the genes encoding $\beta$-tubulin, and elongation factor 1 (EF1) from the P6 strain and those from A. melanogenum P16 were only 80.18, 88.51, 76.87, and $70.07 \%$, respectively, demonstrating that all the similarities were below 95\%. This meant that the P6 strain was an undescribed species of Aureobasidium spp.

Since single-gene/protein sequences contain limited phylogenetic information, concatenation of multiple single-copy and functionally conserved orthologous sequences, single- 


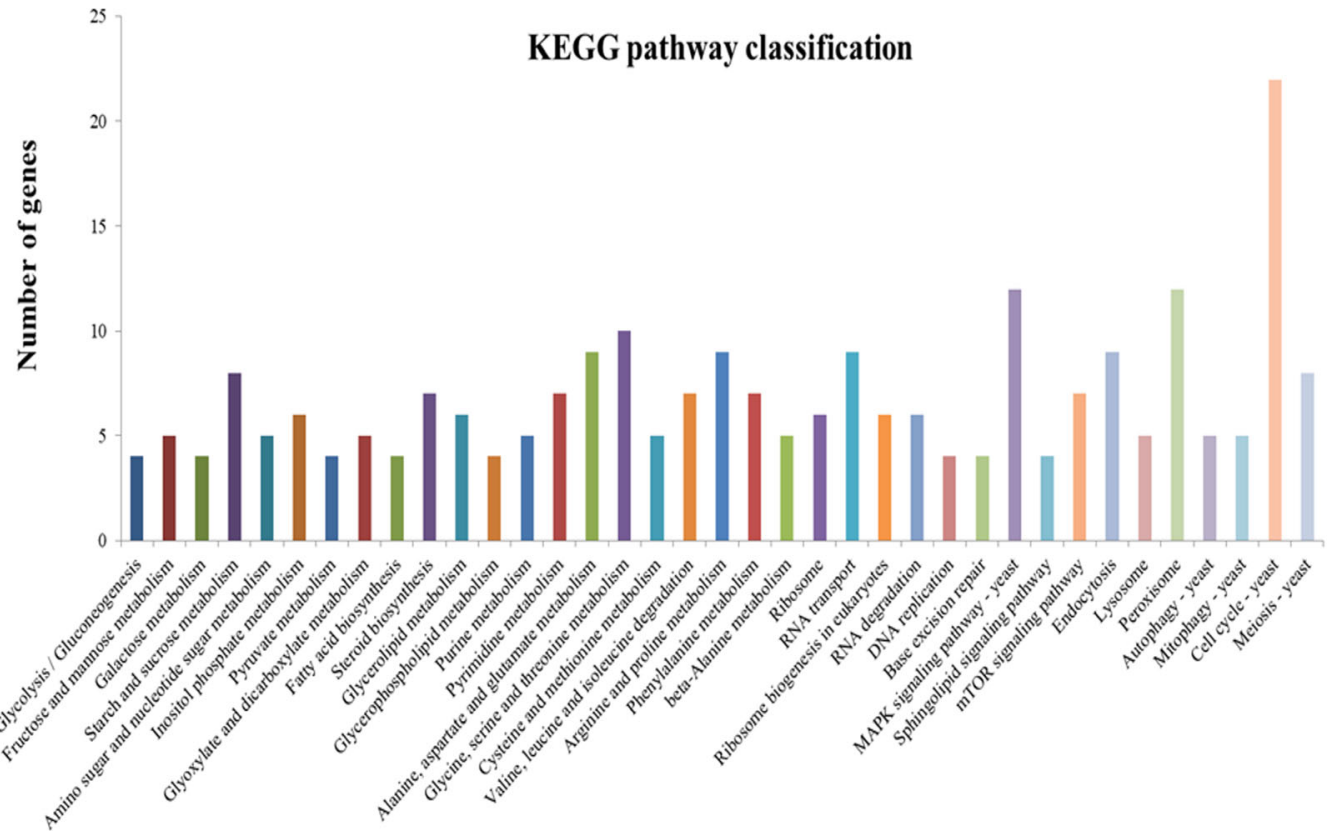

Fig. 2 KEGG pathway classification of the proteins deduced from the genome of the yeast-like fungal strain P6
-Glycolysis / Gluconeogenesis - Fructose and mannose metabolism - Galactose metabolism - Starch and sucrose metabolism - Amino sugar and nucleotide sugar metabolism Inositol phosphate metabolism Pynuate metabolism - Glyoxy late and dicarboxylate metabolism - Fatty acid biosynthesis - Steroid biosynthesis - Glycerolipid metabolism - Glycerophospholipid metabolism - Purine metabolism Purine metabolism
Pyrimidine metabolism - Pyrimidine metabolism
-Alanine, aspartate and glutamate metabolism - Glycine, serine and threonine metabolism - Cysteine and methionine metabolism - Valine, leucine and isoleucine degradation - Arginine and proline metabolism - Phenylalanine metabolism = beta-Alanine metabolism Ribosome
$\square$ Ristanine Ribosome
RNA transport $=$ RNA transport
$=$ Ribosome biogenesis in eukaryotes - RNA degradation -DNA replication = Base excision repair =MAPK signaling pathway - yeast "Sphingolipid signaling pathway $=$ mTOR signaling pathway Endocytosis =Lysosome - Peroxisome =Autophagy - yeast = Mitophagy - yeast Cell cycle - yeast Meiosis - yeast copy orthologs among taxa are commonly used to achieve robust phylogenetic reconstruction with high confidence and concordance (Simao et al. 2015). Therefore, the orthologous genes in the genomes from different strains of Aureobasidium spp. using the GET_HOMOLOGUES v3.2.1
(Contrerasmoreira and Vinuesa 2013) based on the OrthoMCL and BDBH methods were searched and analyzed. The single-copy orthologous genes were combined, and the phylogenetic tree of the single copy orthologous genes was constructed as described above. The results in Fig. 3e

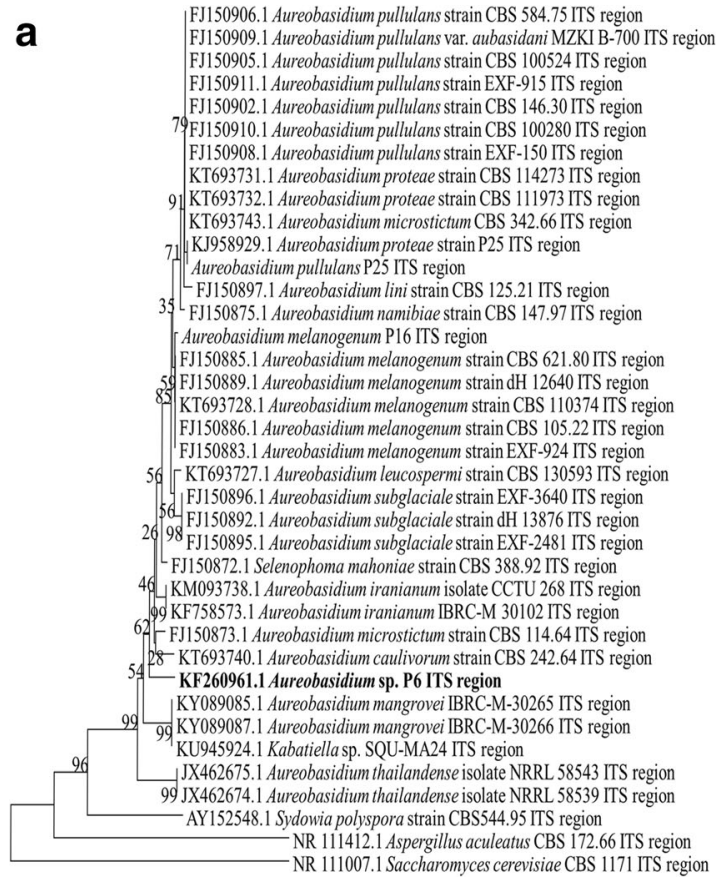

Fig. 3 Neighbor-joining phylogenic analysis of ITS (a) and 28S rDNA (b) regions, the genes encoding EF1 (c), $\beta$-tubulin (d), and the singlecopy orthologous genes (e) showing the relationship of the yeast-like
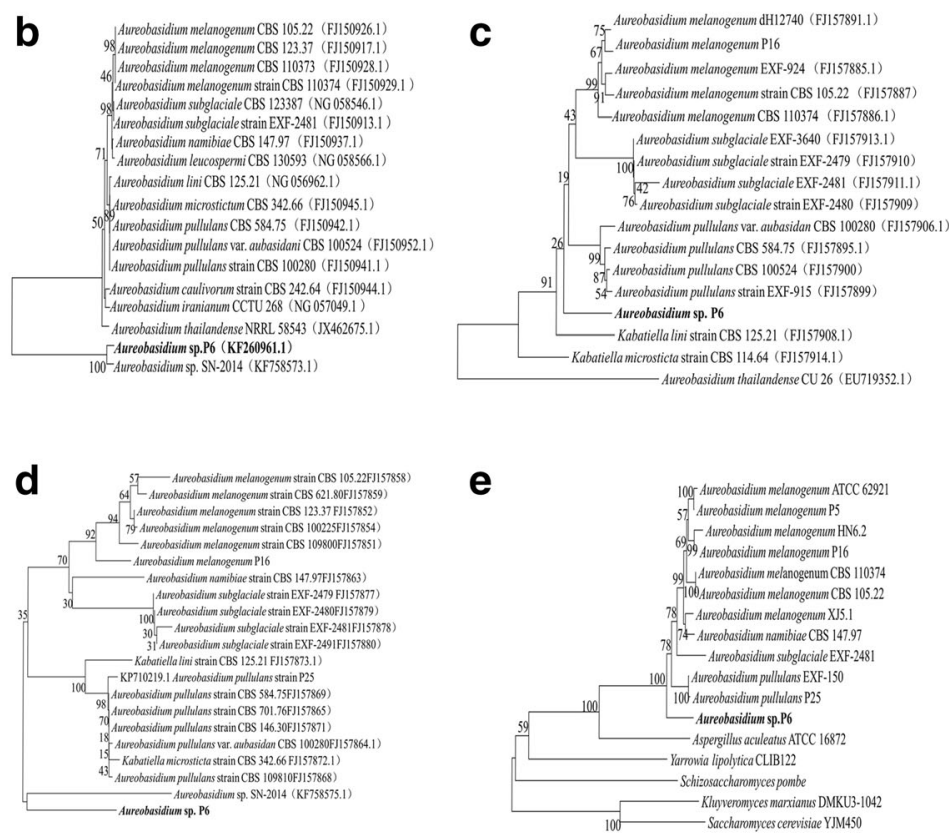

fungal strain P6 with the related taxa. The numbers given on the branches were the frequencies with which a given branch appeared in 1000 bootstrap replications. Bar, 0.020 substitutions per nucleotide position 
demonstrated that the strain P6 was not related to any known species of Aureobasidium spp. by forming a separate branch in the phylogenetic trees, either.

The phylogenetic tree (Fig. 4a) of the whole genomic DNAs from the yeast-like fungal strain P6, A. melanogenum EXF-3378 (Gostinčar et al. 2014), A. melanogenum P5 (Liu et al. 2014), A. melanogenum P16 (Ma et al. 2014), A. subglaciale EXF-2481 (Gostinčar et al. 2014), A. pullulans EXF-150 (Gostinčar et al. 2014), and A. pullulans P25 (Zhao et al. 2019) and the phylogenetic tree of multigene sequences including the ITS, the genes encoding EF-1a, and $\beta$-tubulin sequences (Fig. 4b) also showed that the yeast-like fungal strain P6 was not related to any known species of Aureobasidium spp. by forming a separate branch in the phylogenetic trees (Fig. 4).

Finally, based on its unique DNA sequence and taxonomic position in the phylogenetic trees, the yeast-like fungal strain P6 was identified as Aureobasidium hainanensis sp. nov. P6 isolate.

\section{Divergence time of $A$. hainanensis sp. nov. P6 within the genus of Aureobasidium spp. during evolution}

Accurate estimation of divergence time is essential to understand many evolutionary processes. It has been well known that during evolution ITS sequences in different eukaryotic species were highly conserved (Kurtzman and Fell 2000). Therefore, ITS sequences in different species of Aureobasidium spp. can be used to estimate divergence time of A. hainanensis sp. nov. P6 and other species separation of Aureobasidium spp. Based on the reference phylogenetic tree, we chose the divergence time of 590 Mya

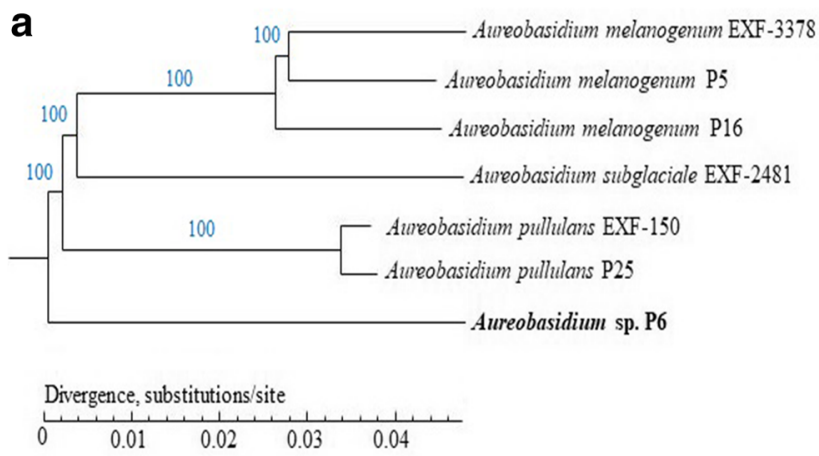

Fig. 4 The genome-wide phylogenetic tree (a) based on the genomes of the yeast-like fungal strain P6 (accession number: RZIQ01000000) and other Aureobasidium spp. strains was constructed by the TreeBeST using the method of a PhyML with the setting bootstraps of 1000, and the number of bootstraps for each node was shown. The tree was displayed to scale, with branch lengths measured in the number of substitutions per site. The accession numbers of all the yeast-like fungal genomes are for S. cerevisiae and A. aculeatus (Fig. 5a). As described in the "Materials and methods," these analyses were performed to estimate the divergence time based on the ITS sequences of different species of Aureobasidium spp. The results in Fig. 5b showed that $S$. cerevisiae and all the strains of Aureobasidium spp. and Aspergillus spp. had a common ancestor 590 Mya ago and A. hainanensis sp. nov. P6 isolate used in this study was evolved to be a separate branch around 23.76 Mya ago, and at that time, it had a common ancestor with the modern-day A. mangroveri $\mathrm{sp}$. nov which was discovered by Nasr et al. (2018) and A. thailandense isolated by Peterson et al. (2013). At the same time, the yeast-like fungal strain P6 also had a common ancestor with any other modern-day species of Aureobasidium spp. reported so far (Fig. 5). However, the divergence time of any other species of Aureobasidium spp. was later than that of A. hainanensis sp. nov. P6, A. mangroveri sp. nov. and A. thailandense. For example, about 14.72 Mya ago A. subglaciale became an offspring; Around 9.48 Mya ago, A. melanogenum that was the most widely distributed in different environments became a separate branch; 7.27 Mya ago A. namibiae strain and A. lini strain became an isolated cluster. The results in Fig. 5 also indicated that A. pullulans and A. proteae which were the most closely phylogenetic appeared only 3.77 Mya ago in the world among all the species of Aureobasidium spp., and they were the youngest branch in the Aureobasidium spp. family. Our data also indicated that the genomes of all strains of A. melanogenum, A. pullulans, A. nambiae, and A. subglaciale carried a PUL1 gene encoding pullulan synthase involved in pullulan biosynthesis while A. hainanensis sp. nov. P6 isolate did not have such a gene, suggesting that the PUL1 gene was gained by any other

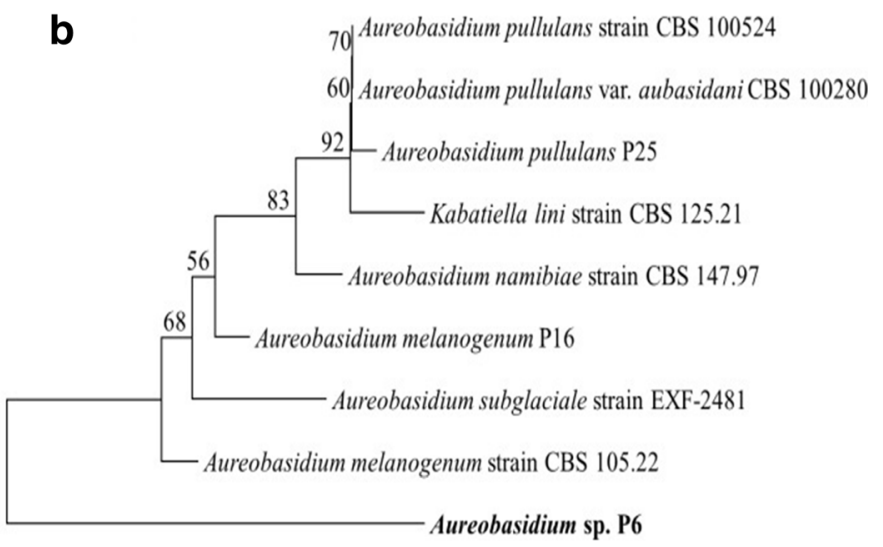

shown in Supplementary file 2. The phylogenetic tree (b) of the yeastlike fungal strain P6 and other strains of Aureobasidium spp. based on a multigene analysis of the ITS sequences; EF- $1 \alpha$ and $\beta$-tubulin obtained from their genomic DNAs was constructed by the neighbor-joining method with 1000 bootstrap replicates using MEGA 7.0.14 software, and Bootstrap values (1000 pseudoreplications) were $\geq 71 \%$ 


\section{a}

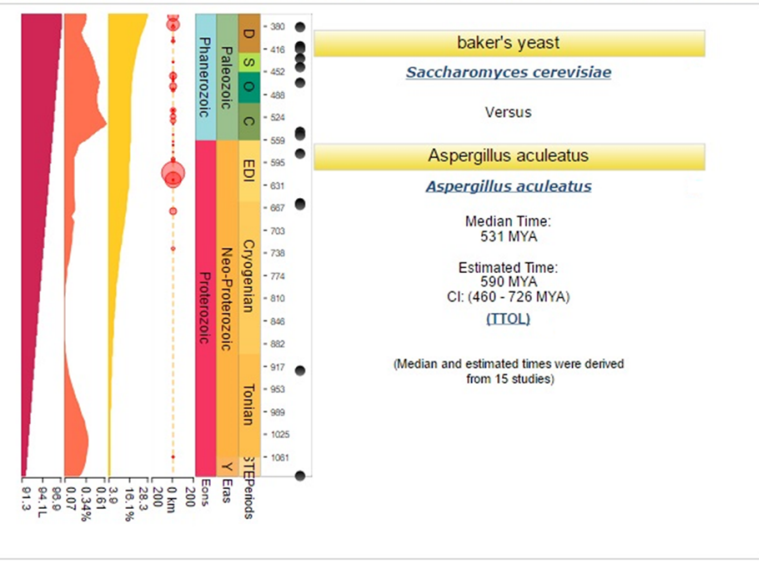

b

FJ150905.1_Aureobasidium pullulans_strain_CBS_100524_ITS region

FJ150908.1_Aureobasidium_pullulans_strain_EXF-150_ITS_region

FJ150911.1_Aureobasidium_pullulans_strain_EXF-915_ITS_region

FJ150906.1_Aureobasidium pullulans_strain_CBS 584.75 ITS region

FJ150902.1_Aureobasidium pullulans_strain_CBS_146.30_ITS_region

KT693732.1_Aureobasidium proteae strain_CBS 111973 ITS region

FJ150910.1_Aureobasidium pullulans_strain_CBS_100280_ITS_region

KT693743.1_Aureobasidium microstictum_CBS 342.66_ITS region

KT693731.1_Aureobasidium proteae_strain_CBS_114273_ITS_region

3.77. FJ150909.1_Aureobasidium_pullulans_var._aubasidani_MZKKI_B-700_ITS_region

KJ958929.1_Aureobasidium proteae strain P25 ITS region

Aureobasidium pullulans_P25_ITS_region

FJ150897.1 Aureobasidium lini strain CBS 125.21 ITS region

7.27. FJ150875.1_Aureobasidium namibiae_strain_CBS_147.97_ITS region

KT693728.1_Aureobasidium melanogenum_strain_CBS_110374_ITS_region

FJ150885.1_Aureobasidium_melanogenum_strain_CBS_621.80_ITS_region FJ150889.1_Aureobasidium_melanogenum_strain_dH_12640_TTS_region 政

FJ150883.1_Aureobasidium_melanogenum_strain_EXF-924_ITS_region FJ150886.1 Aureobasidium melanogenum strain CBS 105.22 ITS region FJ150895.1_Aureobasidium_subglaciale__train_EXF-2481_ITS_region

14.72 FJ150892.1_Aureobasidium_subglaciale_strain_dH_13876_ITS_region

FJ150896.1_Aureobasidium_subglaciale_strain_EXF-3640_ITS_region KT693727.1_Aureobasidium_leucospermi_strain_CBS_130593_TTS_region

KF758573.1 Aureobasidium iraniamum IBRC-M 30102 ITS region

18.20 KM093738.1_Aureobasidium_iranianum_isolate_CCTU_268_ITS_region

FJ150872.1 Selenophoma mahoniae strain CBS 388.92 ITS region

76 [ KT693740.1_Aureobasidium_caulivorum_strain_CBS_242.64_ITS_region

76 [ FJ150873.1_Aureobasidium_microstictum_strain_CBS_114.64_ITS_region

— KF260961.1_Aureobasidium_sp.P6_ITS_region

29.61 KU945924.1_Kabatiella_sp.SQU-MA24_ITS_region

KY089085.1_Aureobasidium mangrovei_IBRC-M-30265 ITS region

KY089087.1_Aureobasidium_mangrovei_IBRC-M-30266_ITS_region

JX462675.1 Aureobasidium thailandense isolate NRRL 58543 ITS region

JX462674.1_Aureobasidium_thailandense_isolate_NRRL_58539_ITS_region

AY152548.1_Sydowia_polyspora_strain_CBS544.95_ITS_region

NR_111412.1_Aspergillus_aculeatus_CBS_172.66_ITS_region

590

NR_111007.1_Saccharomyces_cerevisiae_CBS_1171_ITS_region

Fig. 5 The divergence time (590 Mya) for S. cerevisiae and A. aculeatus (a) and divergence time estimation of different strains of Aureobasidium spp. based on ITS sequences (b)

strains of Aureobasidium spp. during the evolution (data not shown). That was why other species of Aureobasidium spp. especially A. melanogenum can produce a large amount of pullulan (Ma et al. 2014; Jiang et al. 2018; Xue et al. 2019) while A. hainanensis sp. nov. P6 isolate only produced a small amount of unknown exopolysaccharides (Ma et al. 2013). We also discovered that both A. hainanensis sp. nov. P6 isolate and A. pullulans $\mathrm{P} 25$ had high glucose oxidase activity (Ma et al. 2018; Zhao et al. 2019), suggesting that in both A. hainanensis sp. nov. P6 isolate and A. pullulans P25 high glucose oxidase activity was maintained during the evolution. Indeed, the glucose oxidase (GOD1 genes) genes in both $A$. hainanensis sp. nov. P6 isolate and A. pullulans $\mathrm{P} 25$ were very closely matched each other (data not shown). That was why A. hainanensis sp. nov. P6 isolate and A. pullulans P25 can be well applied to produce high level of gluconic acid which has many potential applications (Ma et al. 2018; Zhao et al. 2019). Indeed, the ITS sequences of 38 described species of Enallagma spp. the damselflies were also used to estimate divergence time (Callahan and McPeek 2016).

\section{Evolutional analysis of some key enzymes in $A$. hainanensis sp. nov. $\mathrm{P} 6$ isolate}

It has been well documented that different strains of Aureobasidium spp. can produce amylase, protease, glucose oxidase, esterase, lipase, fructosyltransferase, cellulase, and xylanase (Chi et al. 2009a, b; Aung et al. 2019; Zhao et al. 2019) which are involved in hydrolysis of extracellular nutrients and metabolisms. During the evolution, the enzymes encoding genes must be changed to make them well adapt to the changing environments. The search for them in the genome of A. hainanensis sp. nov. P6 isolate found that it indeed harbored all the genes and the enzymes encoded by them had potential applications. In the phylogenetic trees, all glucoseamylase, alkaline serine protease, esterase, lipase, fructosyltransferase, cellulase, and xylanase from A. hainanensis sp. nov. P6 produced the isolated groups, respectively, meaning that $A$. hainanensis sp. nov. P6 isolate was indeed far related to any other species of Aureobasidium spp. and had unique taxonomic position (Supplementary file 3 ). However, the glucose oxidase encoding genes from both A. hainanensis sp. nov. P6 isolate and A. pullulans $\mathrm{P} 25$ strain 


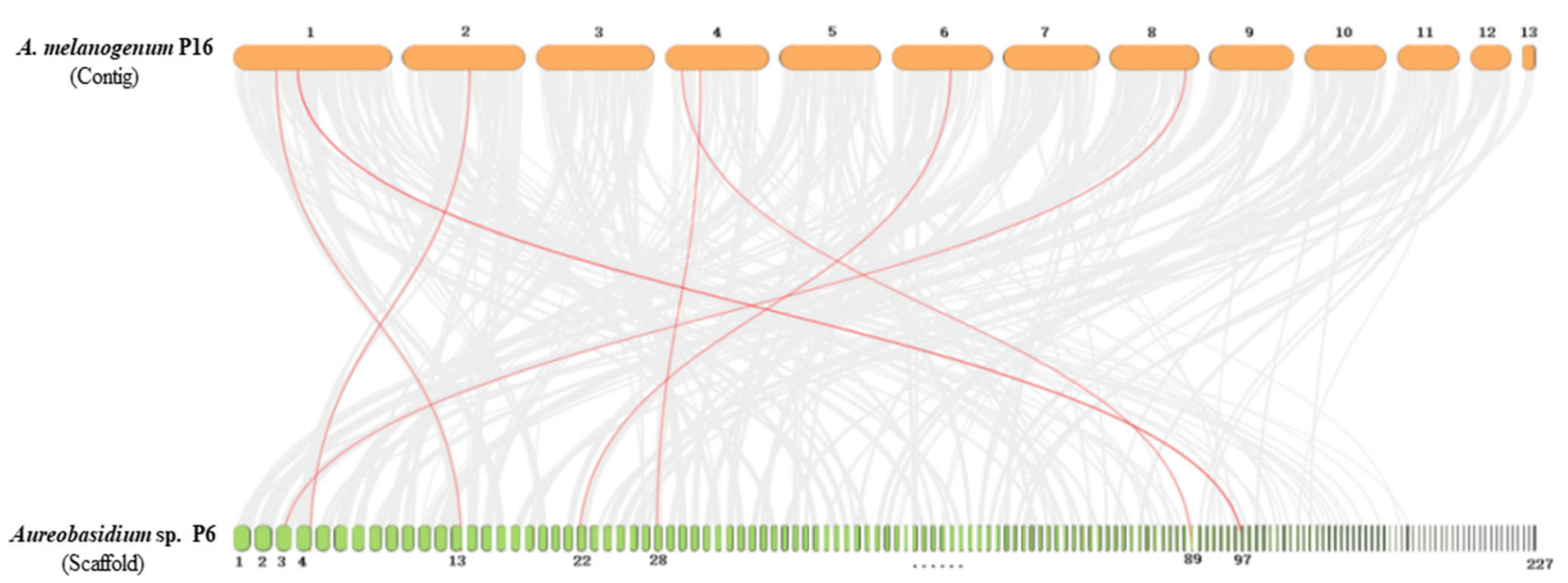

Fig. 6 Collinearity analysis of the P16 strain genome and the P6 strain genome

were very closely matched each other (Supplementary file $3 a$ ). Indeed, both of them have high glucose oxidase activity and could produce high levels of gluconic acid during the fermentation (Ma et al. 2018; Zhao et al. 2019). Collinearity analysis of the P16 strain genome and P6 strain genome showed that glucose oxidase (P6GA4578), $\beta$-glucosidase (P6GA3708), glycoside hydrolase (P6GA5178), $\beta$-xylanase (P6GA0897), and glucoamylase (P6GA4151) genes in A. hainanensis sp. nov. P6 isolate were unique (Fig. 6). This meant that A. hainanensis sp. nov. P6 isolate and their enzymes indeed had some unique application in biotechnology.

In genetics, $\mathrm{Ka} / \mathrm{Ks}$ or $\mathrm{dN} / \mathrm{dS}$ represents ratio of the nonsynonymous base substitution to the synonymous base substitution in the protein encoding gene and can be used to evaluate selective pressure. Any base mutation that cannot cause amino acid change is called the synonymous base substitution while any base mutation that can cause amino acid change is called the non-synonymous base substitution. In general, the synonymous base substitution is not caused by natural selection while the non-synonymous base substitution is caused by natural selection. So $\mathrm{dN} / \mathrm{dS}>1$ means positive selection whereas $\mathrm{dN} / \mathrm{dS}=1$ means neutral selection and $\mathrm{dN} / \mathrm{dS}<1$ means purify selection (Lynch and Conery 2000). In order to show this, the glucose oxidase genes from different strains of Aureobasidium spp. were analyzed using the PAML 4.9 (Yang 2007). The results showed that omega (dN/dS) value of the glucose oxidase gene from A. hainanensis sp. nov. P6 isolate was only 0.06969 and $\mathrm{dN} / \mathrm{dS}$ value was less than 0.25

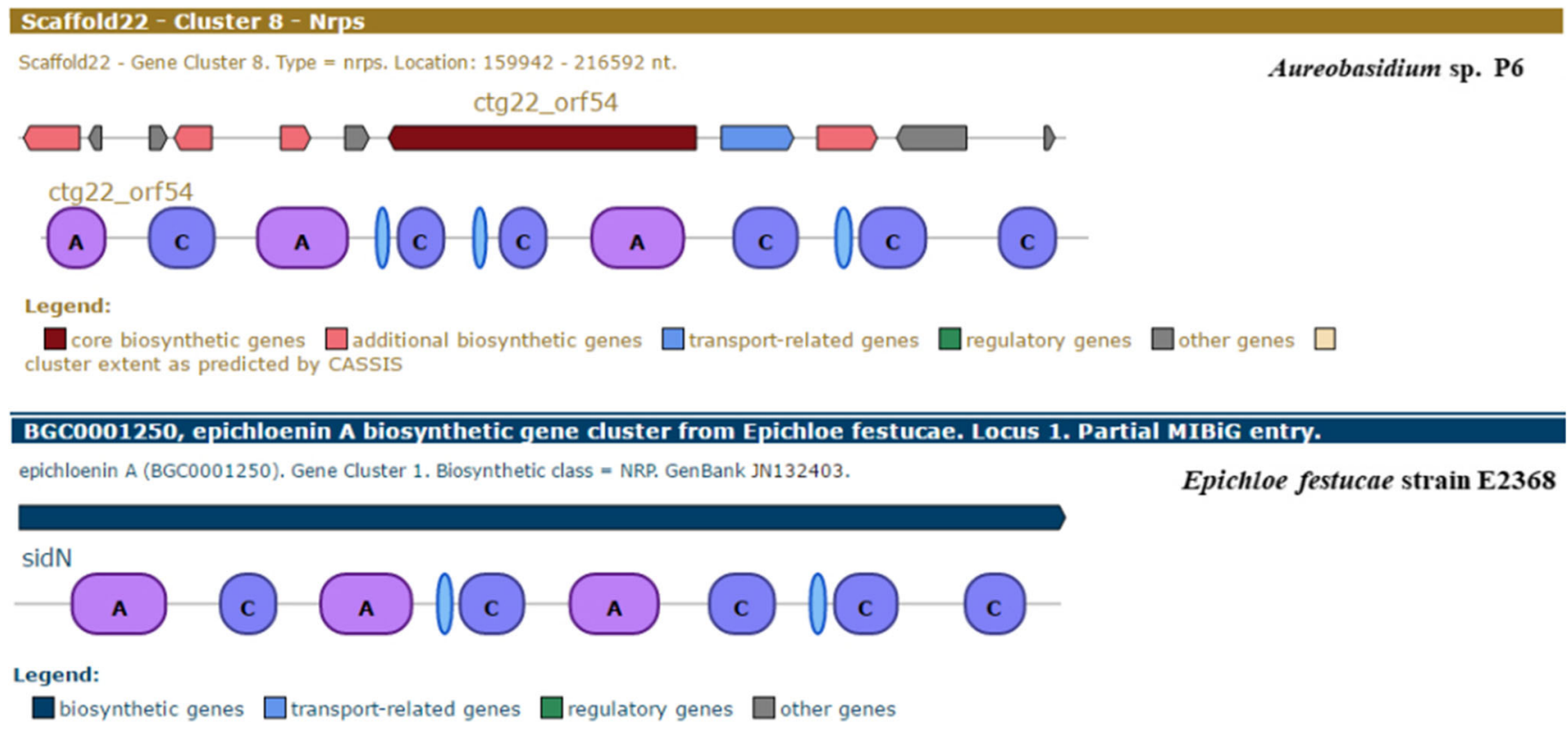

Fig. 7 Domains of the Nrps encoded by the genome of the yeast-like fungal strain P6 and those of the Nrps encoded by the genome of $E$. festucae strain E2368 
(data not shown), suggesting that the glucose oxidase in A. hainanensis sp. nov. P6 genome was purely selected during the evolution to keep its high glucose oxidase activity.

Our data showed that the upstream of the glucose oxidase gene from A. hainanensis sp. nov. P6 isolate had a TATA-box, but did not have a binding site for Mig1, a key repressor in glucose repression, suggesting that biosynthesis of the glucose oxidase responsible for formation of gluconic acid was not repressed by high concentration of glucose in the medium so that high concentration of gluconic acid was produced by A. hainanensis sp. nov. P6 isolate (Ma et al. 2018). The promoter ( $-845 \mathrm{bp})$ of the glucose oxidase gene from A. hainanensis sp. nov. P6 isolate also had a binding site (TTACTAA) for the transcriptional activator YAP1 which is required for oxidative stress and activated by $\mathrm{H}_{2} \mathrm{O}_{2}$ formed during gluconic acid biosynthesis (Ma et al. 2018). The activated YAP1 may be translocated to nuclear to make the cells adapt to the oxidative stress environment with $\mathrm{H}_{2} \mathrm{O}_{2}$ produced during gluconic acid biosynthesis. The promoter ( $-548 \mathrm{bp}$ ) of the glucose oxidase genes from only A. hainanensis sp. nov. P6 strain and A. pulluans P25 strain also had a binding site (TGCCAAG) for the transcriptional activator Rim101p which was similar to the transcriptional activator $\mathrm{PacC}$ in S. cerevisiae. The transcriptional activator Rim $101 \mathrm{p}$ was activated at alkaline or neutral environment through a $\mathrm{pH}$ signal pathway and the activated Rim101p may promote expression of some genes in response to alkaline or neutral surrounding. Indeed, it has been well known that during gluconic acid production, $\mathrm{CaCO}_{3}$ should be added to the fermentation medium to make a neutral environment in order to improve glucose oxidase activity and gluconic acid biosynthesis (Ma et al. 2018; Zhao et al. 2019). This might be related to activation of the transcriptional activator Rim101p and promoted expression of the genes responsible for gluconic acid biosynthesis.

\section{Analysis of the potential secondary metabolites produced by $A$. hainanensis sp. nov. P6 isolate}

Table 1 showed that the genome of A. hainanensis sp. nov. P6 isolate contained three NRPS clusters, five type-I PKS clusters, and one type-I NRPS/PKS cluster, suggesting that it had high potential to biosynthesize different secondary metabolites. Epichloenin A is a new type of siderophore and can significantly promotes iron assimilation in plants and plays a

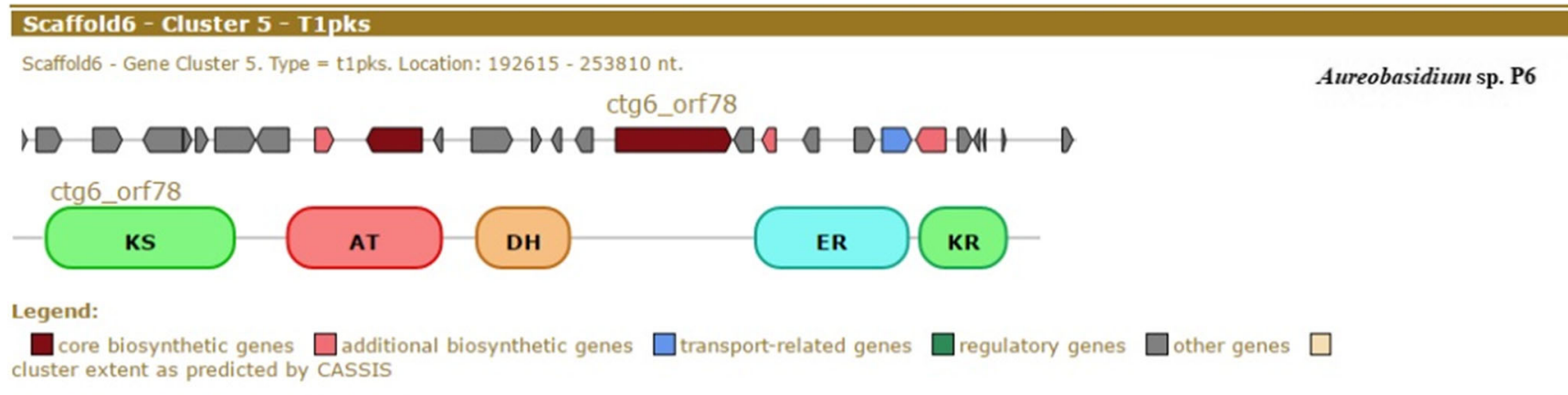

\section{Scaffold36 - Cluster 10 - T1pks}

Scaffold36 - Gene Cluster 10. Type = t1pks. Location: $1-38377 \mathrm{nt}$.

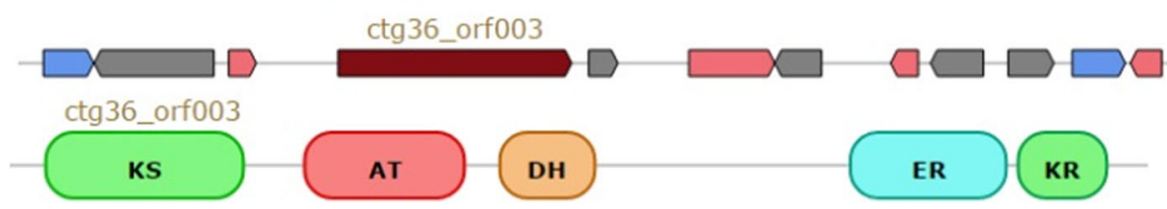

Legend:

$\square$ core biosynthetic genes $\square$ additional biosynthetic genes $\square$ cluster extent as predicted by CASSIS

transport-related genes $\square$ regulatory genes $\square$ other genes BGC0001190, fusaric acid biosynthetic gene cluster from Fusarium verticillioides. Locus 1. Partial MIBiG entry.

fusaric acid (BGC001190). Gene Cluster 1. Biosynthetic class = Polyketide. GenBank CM000580, positions 756250-809209.

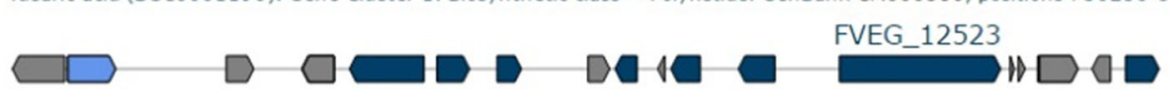

FVEG_12523
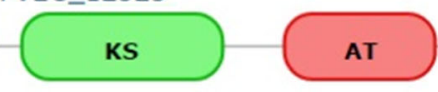

\section{DH}

ER

Legend:

$\square$ biosynthetic genes $\square$ transport-related genes $\square$ regulatory genes $\square$ other genes

Fig. 8 Domains of T1Pks encoded by the Cluster 5 and the Cluster 10 in the genomes of the yeast-like fungal strain P6 and F. verticillioides 7600 


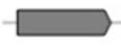

\section{$\mathrm{CD}$}

\section{$\square$}

ctg18_orf5

KS

\section{AT}

\section{ACP $\mathrm{ACP} \quad T$}

Legend:

$\square$ core biosynthetic genes $\square$ additional biosynthetic genes $\square$ transport-related genes $\square$ regulatory genes $\square$ other genes cluster extent as predicted by CASSIS

\section{Scaffold118 - Cluster 18 - T1pks}

Scaffold118 - Gene Cluster 18. Type $=$ t1pks. Location: $1-38375$ nt.

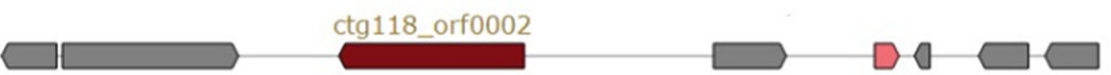

ctg118_orf0002

KS

AT

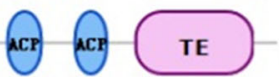

Legend:

$\square$ core biosynthetic genes $\square$ additional biosynthetic genes $\square$ transport-related genes $\square$ regulatory genes $\square$ other genes $\square$ cluster extent as predicted by CASSIS

\section{BGC0001272, elsinochrome B / elsinochrome C biosynthetic gene cluster from Elsinoe fawcettii. Locus 1. Partial MIBiG entry.}

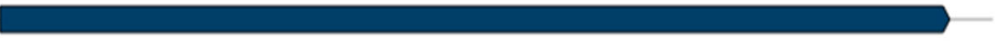

PKS1

\section{AT}

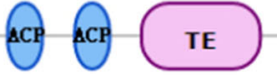

\section{Legend:}

$\square$ biosynthetic genes $\square$ transport-related genes $\square$ regulatory genes $\square$ other genes

Fig. 9 Domains of the T1Pkss encoded by the Cluster 7 and the Cluster 18 in the genome of the yeast-like fungal strain P6 and Pks 1 encoded by the genome of E. fawcettii

\section{Scaffold101 - Cluster 16 - T1pks-nrps}

Scaffold101 - Gene Cluster 16. Type = t1pks-nrps. Location: $15604-70195 \mathrm{nt}$.

Aureobasidium sp. P6

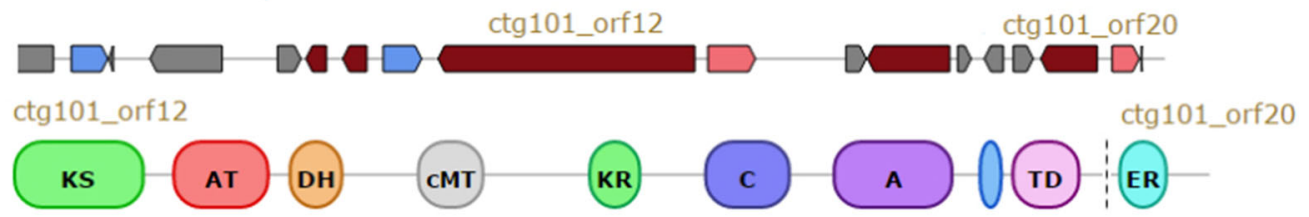

Legend: $\square$ core biosynthetic genes $\square$ additional biosynthetic genes $\square$ transport-related genes $\square$ regulatory genes $\square$ other genes
cluster extent as predicted by CASSIS

\section{BGC0000992, fusaridione A biosynthetic gene cluster from Fusarium heterosporum. Locus 1. Partial MIBiG entry.}

fusaridione A (BGC0000992). Gene Cluster 1. Biosynthetic class = NRP/Polyketide. GenBank AY700570

Fusarium heterosporum strain ATCC 74349

$\square \square \square \square \square \square \square \square \square \square \square \square \square$

fsds

KS

AT

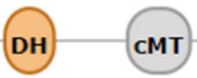

KR
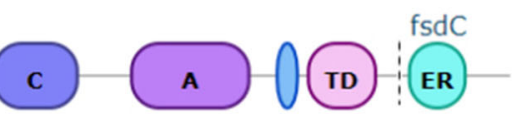

Legend:

$\square$ biosynthetic genes $\square$ transport-related genes $\square$ regulatory genes $\square$ other genes

Fig. 10 Domains of the Pks-Nrps encoded by the Cluster 16 in the genome of the yeast-like fungal P6 and those of the Pks-Nrps in F. heterosporum strain ATCC74349 
critical role in fungal-host relationships. Analysis of the genome of A. hainanensis sp. nov. P6 isolate found a NRPS gene cluster encoding domain $\mathrm{A}$, domain $\mathrm{T} / \mathrm{PCP}$ and domain $\mathrm{C}$ (Fig. 7). The domains encoded by the NRPS gene cluster were the same as those encoded by a SidN (JN132403.1) in Epichloe festucae strain E2368 (Johnson et al. 2013) (Fig. 7). The NRPS gene cluster in E. festucae strain E2368 has been demonstrated to catalyze biosynthesis of extracellular epichloenin A, one kind of ferritin family (Johnson et al. 2013). This meant that A. hainanensis sp. nov. P6 isolate used in this study could also produce such an epichloenin A. It has also been reported that $A$. melanogenum and $A$. namibiae have one putative synthase for siderophores, whereas A. subglaciale has two copies, and A. pullulans has three copies (Gostinčar et al. 2014). Indeed, A. melanogenum HN6.2 strain can produce cyclic fusigen, linear fusigen, ferricrocin, and hydroxyferricrocin (Lu et al. 2019).

Search for the PKS gene cluster in the genome of A. hainanensis sp. nov. P6 isolate identified two T1pkss (a Cluster 5 and a Cluster 10) which domains were very similar to those of the Pks in Fusarium verticillioides 7600 that could produce fusaric acid (Brown et al. 2014) (Fig. 8). Fusaric acid is a picolinic acid derivative which likely inhibits dopamine $\beta$-hydroxylase, cell proliferation, DNA synthesis, and quorum sensing.

The T1Pkss encoded by a Cluster 7 and a Cluster 18 in the genome of A. hainanensis sp. nov. P6 isolate had AT domain, KS domain, ACP domain, and TE domain which were the same as those of the Pks1 encoded by the genome of Elsinoë fawcettii that produced elsinochromes (Chung and Liao 2008) (Fig. 9). Elsinochromes are the non-hostselective toxins which react with oxygen molecules after light activation to produce highly toxic reactive oxygen species. They have photosensitive and antitumor activities and appear to be coordinately regulated by light, nutrients, and $\mathrm{pH}$. They have a good resistance to pests and diseases with no sideeffects on human body. Besides that, elsinochrome A has the highest quantum yield of singlet oxygen and is known as a good photosensitizer in visible region (Meille et al. 1989), which is a natural drug with great development value.

The T1Pks-Nrps encoded by a Cluster 16 in the genome of A. hainanensis sp. nov. P6 isolate may be responsible for biosynthesis of fusaridione because the domains of the T1Pks-Nrps were similar to those of the Pks-Nrps in Fusarium heterosporum strain ATCC74349 which has been demonstrated to produce fusaridione (Kakule et al. 2013) (Fig. $10)$. Fusaridione $\mathrm{A}$ is a novel tyrosine-derived 2,4pyrrolidinedione produced by a number of Fusarium species with antimicrobial and cytotoxic activity. This bright yellow compound may display an inhibitory activity against mitochondrial ATPases and specifically inhibit the substrate anion carriers of the inner membrane of the mitochondria (Kakule et al. 2013).

\section{Conclusions}

All the results mentioned above clearly indicated that the genome of Aureobasidium sp. P6 strain was completely different from those of any other species of Aureobasidium spp.; this strain was finally identified as a new species $A$. hainanensis sp. nov. P6 of Aureobasidium spp. and had unique taxonomic and evolutional positions. It also may produce different kinds of bioactive secondary metabolites and enzymes, suggesting that it had highly potential applications in biotechnology. However, it should be tested whether or not it could synthesize such bioactive secondary metabolites by experiments.

Funding This research work was supported by the National Natural Science Foundation of China (Grant No. 31770061).

\section{Compliance with ethical standards}

Conflict of interest The authors declare that they have no conflict of interest.

Ethical approval This article does not contain any studies with human participants or animals performed by any of the authors.

Informed consent Informed consent was obtained from all individual participants included in the study.

Open Access This article is distributed under the terms of the Creative Commons Attribution 4.0 International License (http:// creativecommons.org/licenses/by/4.0/), which permits unrestricted use, distribution, and reproduction in any medium, provided you give appropriate credit to the original author(s) and the source, provide a link to the Creative Commons license, and indicate if changes were made.

\section{References}

Altschul SF, Gish W, Miller W, Myers EW, Lipman DJ (1990) Basic local alignment search tool. J Mol Biol 215(3):403-410

Aung T, Jiang H, Chen CC, Liu GL, Hu Z, Chi ZM, Chi Z (2019) Production, gene cloning, and overexpression of a laccase in the marine-derived yeast Aureobasidium melanogenum strain 11-1 and characterization of the recombinant laccase. Mar Biotechnol 21:7687

Besemer J, Lomsadze A, Borodovsky M (2001) GeneMarkS: a selftraining method for prediction of gene starts in microbial genomes. Implications for finding sequence motifs in regulatory regions. Nucleic Acids Res 29(12):2607-2618

Blin K, Medema MH, Kazempour D, Fischbach MA., Breitling R, Takano E, Weber T (2013) antiSMASH 2.0 - a versatile platform for genome mining of secondary metabolite producers. Nucle Acids Res gkt449

Brown DW, Lee SH, Kim LH, Ryu JE, Lee S, Seo Y, Kim YH, Busman M, Yun SH, Proctor RH, Lee T (2014) Identification of a 12-gene fusaric acid biosynthetic gene cluster in Fusarium species through comparative and functional genomics. Mole Plant-Microb Interact 28(3):319-332 
Callahan MS, McPeek MA (2016) Multi-locus phylogeny and divergence time estimates of Enallagma damselflies (Odonata: Coenagrionidae). Mole Phylog Evol 94:182-195

Cañete-Rodríguez AM, Santos-Dueñas IM, Jiménez-Hornero JE, Ehrenreich A, Liebl, Garcia-Garcia I (2016) Gluconic acid: Properties, production methods and applications - an excellent opportunity for agro-industrial by-products and waste bio-valorization. Process Biochem 51 (12): S1359511316303476

Capella-Gutiérrez S, Silla-Martínez JM, Gabaldón T (2009) trimAl: A tool for automated alignment trimming in large-scale phylogenetic analyses. Bioinform 25(15):1972-1973

Chan GF, Bamadhaj MH, Gan HM, Rashid NAA (2012) Genome sequence of Aureobasidium pullulans AY4, an emerging opportunistic fungal pathogen with diverse biotechnological potential. Eukaryot Cell 11(11):1419-1420

Chen X, Wang QQ, Liu NN, Liu GL, Chi Z, Chi ZM (2017a) A glycosyltransferase gene responsible for pullulan biosynthesis in Aureobasidium melanogenum P16. Int J Biol Macromol 95:539

Chen Y, Chen Y, Shi C, Huang Z, Zhang Y, Li S, Li Y, Ye J, Yu C, Li Z, Zhang X, Wang J, Yang H, Fang L, Chen Q (2017b) SOAPnuke: A MapReduce acceleration supported software for integrated quality control and preprocessing of high-throughput sequencing data. Gigasci 7(1):1-6

Chi ZM, Chi Z, Zhang T, Liu GL, Li J, Wang XH (2009a) Production, characterization and gene cloning of the extracellular enzymes from the marine-derived yeasts and their potential applications. Biotechnol Adv 27(3):236-255

Chi ZM, Wang F, Chi Z, Yue LX, Liu GL, Zhang T (2009b) Bioproducts from Aureobasidium pullulans, a biotechnologically important yeast. Appl Microbiol Biotechnol 82(5):793-804

Chi Z, Wang XX, Ma ZC, Buzdar MA, Chi ZM (2012) The unique role of siderophore in marine-derived Aureobasidium pullulans HN6.2. Biomet 25(1):219-230

Chi Z, Liu GL, Liu CG, Chi ZM (2016) Poly( $\beta$-1-malic acid) (PMLA) from Aureobasidium spp. and its current proceedings. Appl Microbiol Biotechnol 100(9):3841-3851

Chung KR, Liao HL (2008) Determination of a transcriptional regulatorlike gene involved in biosynthesis of elsinochrome phytotoxin by the citrus scab fungus, Elsinoe fawcettii. Microbiol 154(Pt 11): $3556-3566$

Contrerasmoreira B, Vinuesa P (2013) GET HOMOLOGUES, a versatile software package for scalable and robust microbial pangenome analysis. Appl Environ Microbiol 79(24):7696-7701

Garay LA, Sitepu IR, Cajka T, Xu J, The HE, German JB, Pan ZL, Dungan SR, Block DE, Boundy-Mills KL (2018) Extracellular fungal polyol lipids: a new class of potential high value lipids. Biotechnol Adv 36(2):397-414

Garnica S, Riess K, Schön ME, Oberwinkler F, Setaro SD (2016) Divergence times and phylogenetic patterns of sebacinales, a highly diverse and widespread fungal lineage. PLoS One 11:202-210

Gostinčar C, Ohm RA, Kogej T, Sonjak S, Turk M, Zajc J, Zalar P, Grube M, Sun H, Han J, Sharma A, Chiniquy J, Yee Ngan CY, Lipzen A, Barry K, Grigoriev IV, Gunde-Cimerman N (2014) Genome sequencing of four Aureobasidium pullulans varieties: biotechnological potential, stress tolerance, and description of new species. BMC Genomics 15(1):549-550

Grant JR, Stothard P (2008) The CGView Server: a comparative genomics tool for circular genomes. Nucleic Acids Res 36:W181-W184

Jiang H, Liu NN, Liu GL, Chi Z, Wang JM, Zhang LL, Chi ZM (2016a) Melanin production by a yeast strain XJ5-1 of Aureobasidium melanogenum isolated from the Taklimakan desert and its role in the yeast survival in stress environments. Extremop 20(4):567-577

Jiang H, Ma Y, Chi Z, Liu GL, Chi ZM (2016b) Production, Purification, and gene cloning of a $\beta$-fructofuranosidase with a high inulinhydrolyzing activity produced by a novel yeast Aureobasidium sp.
P6 isolated from a mangrove ecosystem. Mar Biotechnol 18(4):500 510

Jiang H, Xue SJ, Li YF, Liu GL, Chi ZM, Hu Z, Chi Z (2018) Efficient transformation of sucrose into high pullulan concentrations by Aureobasidium melanogenum TN1-2 isolated from a natural honey. Food Chem 257:29-35

Jiang N, Liang YM, Tian CM (2019) Aureobasidium pini sp. nov. from pine needle in China. Phytotaxa 402(4):199-206

Johnson LJ, Koulman A, Christensen M, Lane GA, Fraser K, Forester N, Johnson RD, Bryan GT, Rasmussen S (2013) An extracellular siderophore is required to maintain the mutualistic interaction of Epichloë festucae with Lolium perenne. PLoS Pathog 9(5): e1003332

Kakule TB, Sardar D, Lin Z, Schmidt EW (2013) Two related pyrrolidinedione synthetase loci in Fusarium heterosporum ATCC 74349 produce divergent metabolites. ACS Chem Biol 8(7):1549 1557

Kanehisa M, Goto S, Kawashima S, Okuno Y, Hattori M (2004) The KEGG resource for deciphering the genome. Nucleic Acids Res 32(90001):277D-2280D

Katoh K, Misawa K, Kuma K, Miyata T (2002) MAFFT: a novel method for rapid multiple sequence alignment based on fast Fourier transform. Nucleic Acids Res 30(14):3059-3066

Koonin EV, Fedorova ND, Jackson JD, Jacobs AR, Krylov DM, Makarova KS, Mazumder R, Mekhedov SL, Nikolskaya AN, Rao BS, Rogozin IB, Smirnov S, Sorokin AV, Sverdlov AV, Vasudevan S, Wolf YI, Yin JJ, Natale DA (2004) A comprehensive evolutionary classification of proteins encoded in complete eukaryotic genomes. Genome Biol 5(2):R7

Kumar S, Stecher G, Tamura K (2016) MEGA7: Molecular evolutionary genetics analysis version 7.0 for bigger datasets. Mole Biol Evol 33(7): 1870

Kurtzman CP, Fell JW (2000) The yeasts - a taxonomic study (4th revised and enlarged edition). Amsterdam: Elsevier, pp 222-360

Li HF, Chi ZM, Wang XH, Duan XH, Ma LY, Gao LM (2007) Purification and characterization of extracellular amylase from the marine yeast Aureobasidium pullulans N13d and its raw potato starch digestion. Enzym Microb Technol 40(5):1006-1012

Li RQ, Zhu HM, Ruan J, Qian WB, Fang XD, Shi ZB, Li YR, Li ST, Shan G, Kristiansen K, Li SG, Yang HM, Wang J, Wang J (2010) De novo assembly of human genomes with massively parallel short read sequencing. Genome Res 20(2):265-272

Liu ZQ, Li XY, Chi ZM, Wang L, Li J, Wang XH (2008) Cloning, characterization and expression of the extracellular lipase gene from Aureobasidium pullulans HN2-3 isolated from sea saltern. Antonie Van Leeuwenhoek 94(2):245-255

Liu YY, Chi Z, Wang ZP, Liu GL, Chi ZM (2014) Heavy oils, principally long-chain-alkanes secreted by Aureobasidium pullulans var. melanogenum strain P5 isolated from mangrove system. J Ind Microbiol Biotechnol 41(9):1329-1337

Lu Y, Liu GL, Jiang H, Chi ZM, Chi Z (2019) An insight into the iron acquisition and homeostasis in Aureobasidium melanogenum HN6.2 strain through genome mining and transcriptome analysis. Functional \& Integrative Genom 19(1):137-150

Luo R, Liu B, Xie Y, Li Z, Huang W, Yuan J, He G, Chen Y, Pan Q, Liu Y, Tang J, Wu G, Zhang H, Shi Y, Liu Y, Yu C, Wang B, Lu Y, Han C, Cheung DW, Yiu SM, Peng S, Xiaoqian Z, Liu G, Liao X, Li Y, Yang H, Wang J, Lam TW, Wang J (2012) SOAPdenovo2: an empirically improved memory-efficient short-read de novo assembler. GigaSci 1(1): 18

Lynch M, Conery JS (2000) The evolutionary fate and consequences of duplicate genes. Sci 2290:1151-1155

Ma Y, Wang GY, Liu GL, Wang ZP, Chi ZM (2013) Overproduction of poly ( $\beta$-malic acid) (PMA) from glucose by a novel Aureobasidium sp. P6 strain isolated from mangrove system. Appl Microbiol Biotechnol 97:8931-8939 
Ma ZC, Fu WJ, Liu GL, Wang ZP, Chi ZM (2014) High-level pullulan production by Aureobasidium pullulans var melanogenium P16 isolated from mangrove system. Appl Microbiol Biotechnol 98(11): 4865-4873

Ma Y, Chi Z, Li YF, Jiang H, Liu GL, Hu Z, Chi ZM (2018) Cloning, deletion, and overexpression of a glucose oxidase gene in Aureobasidium sp. P6 for $\mathrm{Ca}^{2+}$-gluconic acid overproduction. Ann Microbiol 68:871-879

Magrane M, Consortium U $(2011,2011)$ UniProt Knowledgebase: a hub of integrated protein data. Database:bar009-bar009

Meille SV, Malpezzi L, Allegra G, Nasini G, Weiss U (1989) Structure of elsinochrome A: a perylenequinone metabolite. Acta Crystallograp Sect C: Crystal Struct Commun 45(4):628-632

Nandi T, Ong C, Singh AP, Boddey J, Atkins T, Sarkartyson M, Essexlopresti AE, Chua HH, Pearson T, Kreisberg JF, Nilsson C, Ariyaratne P, Ronning C, Losada L, Ruan Y, Sung WK, Woods D, Titball RW, Beacham I, Peak I, Keim P, Nierman WC, Tan P (2010) A genomic survey of positive selection in Burkholderia pseudomallei provides insights into the evolution of accidental virulence. PLoS Pathog 6(4):e1000845

Nasr S, Mohammadimehr M, Geranpayeh Vaghei M, Amoozegar MA, Shahzadeh Fazeli SA (2018) Aureobasidium mangrovei sp. nov., an ascomycetous species recovered from Hara protected forests in the Persian Gulf, Iran. Antonie Van Leeuwenhoek 111:1697-1705

Ni X, Chi Z, Ma C, Madzak C (2008) Cloning, characterization and expression of the gene encoding alkaline protease in the marine yeast Aureobasidium pullulans10. Mar Biotechnol 10(3):319-327

Ohm RA, Feau N, Henrissat B, Schoch CL, Horwitz BA, Barry KW, Condon BJ, Copeland AC, Dhillon B, Glaser F, Hesse CN, Kosti I, LaButti K, Lindquist EA, Lucas S, Salamov AA, Bradshaw RE, Ciuffetti L, Hamelin RC, Kema GHJ, Lawrence C, Scott JA, Spatafora JW, Turgeon BG, de Wit PJGM, Zhong S, Goodwin SB, Grigoriev IV (2012) Diverse lifestyles and strategies of plant pathogenesis encoded in the genomes of eighteen Dothideomycetes Fungi. PLoS Pathog 28(12):e1003037

Peterson SW, Manitchotpisit P, Leathers TD (2013) Aureobasidium thailandense sp. nov. isolated from leaves and wooden surfaces. Int J Syst Evol Microbiol 63(Pt 2):790-795

Prabhugaonkar A, Pratibha J (2018) Aureobasidium khasianum (Aureobasidiaceae) a novel species with distinct morphology. Phytotaxa 374 (3:257-262

Prasongsuk S, Lotrakul P, Ali I, Bankeeree W, Hunsa Punnapayak H (2018) The current status of Aureobasidium pullulans in biotechnology. Folia Microbiol 63:129-140

Rambaut A, Drummond AJ, Xie D, Baele G, Suchard MA (2018) Posterior summarisation in Bayesian phylogenetics using Tracer 1.7. System Biol. https://doi.org/10.1093/sysbio/syy032
Rutherford K, Parkhill J, Crook J, Horsnell T, Rice P, Rajandream MA, Barrell B (2000) Artemis: sequence visualization and annotation. Bioinform 16:944-945

Simao FA, Waterhouse RM, Ioannidis P (2015) Kriventseva EV \& Zdobnov EM. BUSCO: assessing genome assembly and annotation completeness with single-copy orthologs. Bioinform btv351

Suchard MA, Lemey P, Baele G, Ayres DL, Drummond AJ, Rambaut A (2018) Bayesian phylogenetic and phylodynamic data integration using BEAST 1.10. Virus Evol 4(1):23-30

Teixeira MC, Monteiro P, Jain P., Tenreiro S, Fernandes AR, Mira NP, Alenquer M, Freitas AT, Oliveira AL, Sa'Correia I (2006) The Yeastract database: a tool for the analysis of transcription regulatory associations in Saccharomyces cerevisiae. Nucleic Acids Res 34 (90001): D446-D451

Wang Y, Tang H, DeBarry JD, Tan X, Li J, Wang X, Lee T, Jin H, Marler B, Guo H, Kissinger JC, Paterson AH (2012) MCScanX: a toolkit for detection and evolutionary analysis of gene synteny and collinearity. Nucleic Acids Res 40(7):e49

Wang CL, Li Y, Xin FH, Liu YY, Chi ZM (2014) Single cell oil production by Aureobasidium pullulans var. melanogenum P10 isolated from mangrove systems for biodiesel making. Process Biochem 49:725-731

Weber T, Blin K, Duddela S, Krug D, Kim HK, Bruccoleri R, Lee SY, Fischbach MA, Muller R, Wohlleben W, Breitling R, Takano E, Medema MH (2015) antiSMASH 3.0 - a comprehensive resource for the genome mining of biosynthetic gene clusters. Nucleic Acids Res 43(W1):W237-W243

Xue SJ, Chen L, Jiang H, Liu GL, Chi ZM, Hu Z, Chi Z (2019) High pullulan biosynthesis from high concentration of glucose by a hyperosmotic resistant, yeast-like fungal strain isolated from a natural comb-honey. Food Chem 286:123-128

Yang Z (2007) PAML 4: Phylogenetic analysis by maximum likelihood. Mol Biol Evol 24(8):1586-1591

Zalar P, Gostincar C, de Hoog GS, Ursic V, Sudhadham M, GundeCimerman N (2008) Redefinition of Aureobasidium pullulans and its varieties. Stud Mycol 61:21-38

Zhang H, Yohe T, Huang L, Entwistle S, Wu P, Yang ZL, Busk PK, Xu Y, Yin YB (2018) dbCAN2: a meta server for automated carbohydrateactive enzyme annotation. Nucle Acids Res 46(W1, 2):W95-W101

Zhao SF, Jiang H, Chi Z, Liu GL, Chi ZM, Chen TJ, Yang G, Hu Z (2019) Genome sequencing of Aureobasidium pullulans P25 and overexpression of a glucose oxidase gene for hyperproduction of $\mathrm{Ca}^{2+}$ gluconic acid. Antonie Van Leeuwenhoek 112:669-678

Publisher's note Springer Nature remains neutral with regard to jurisdictional claims in published maps and institutional affiliations. 